\section{Human Cloning?}

\section{Teil 2: Ist "therapeutisches Klonen» abgrenzbar?}

\section{Rehmann-Sutter}

Im ersten Teil dieser Serie wurde das Projekt "therapeutisches Klonen" unter dem Gesichtspunkt des Embryonenschutzes diskutiert [1]. Es hat sich herausgestellt, dass dabei eine grundsätzlich neue ethische Frage aufgeworfen wird, nämlich die, ob menschliche Embryonen, die aus Kerntransfer entstehen, einen ethischen Sonderstatus haben. Wenn eine Eizelle zuerst entkernt und dann mit einer geeigneten Körperzelle eines Patienten oder einer Patientin fusioniert wird, entsteht zweifellos eine Art menschlicher Embryo, dem vielleicht sogar das Potential innewohnt, sich $\mathrm{zu}$ einem gesunden Kind $\mathrm{zu}$ entwickeln. Dies muss man nach dem Gelingen entsprechender Tierexperimente (z.B. das Schaf Dolly) mindestens annehmen. Ist allein dieses Potential massgeblich für die ethische Zuordnung, wird ein Nukleustransferembryo ethisch und rechtlich gleich behandelt werden müssen wie ein aus Befruchtung entstandener Embryo. Ist zusätzlich aber auch die Entstehungsgeschichte des Embryos massgeblich, d.h. die Umstände seines Entstehens, würde sich vielleicht eine Sonderbehandlung rechtfertigen lassen. Embryonen, die aus einer Körperzelle eines Patienten/einer Patientin mit Hilfe einer vorher entkernten Eizelle hergestellt werden, könnten dann als (externer) Teil des Körpers des Patienten/der Patientin betrachtet werden. Entsprechend müssten die allgemeinen Schutzprinzipien für Embryonen nicht gelockert werden, wenn therapeutisches Klonen erlaubt würde. Wenn die Entstehungsgeschichte eine Rolle spielt, wäre eine Zulassung des therapeutischen Klonens nicht notwendigerweise mit einer Aufweichung der moralischen Standards für den Embryonenschutz verbunden. Diese Frage wurde so identifiziert und dargestellt.

Die einfachste Antwort wäre natürlich folgende: der ganze Zellkern stammt vom Patienten, also gehört der Kerntransferembryo zu seinem Körper. Das impliziert aber die Gleichsetzung vom Zellkern und persönlicher Identität; es ist eine unhaltbare Gleichung, weil sie einen genetischen Reduktionismus enthält. So einfach kann das therapeutische Klonen nicht legitimiert werden.

Nun kann aber schon ganz grundsätzlich gegen die eingeschlagene Argumentationsstrategie eingewendet werden, dass das therapeutische Klonen - wie auch

Korrespondenz:

PD Dr. phil., dipl. biol. Christoph Rehmann-Sutter

Universität Basel

Arbeitsstelle für Bioethik

Institut für Geschichte und Epistemologie der Medizin

Schönbeinstrasse 20

CH-4056 Basel immer man es ethisch einschätzt - nicht abgrenzbar ist vom reproduktiven Klonen. Weil das reproduktive Klonen - also die Klonierung von Menschen als Fortpflanzungstechnologie - überwiegend negativ beurteilt wird, u.a. aus schwerwiegenden kindesrechtlichen Bedenken, könnte dann auch das therapeutische Klonen nicht zugelassen werden. Zwischen therapeutischem und reproduktivem Klonen könnte - so der Einwand - eine Art slippery slope (rutschiger Abhang) bestehen, auf dem es dann kein Halten mehr gäbe. Dies ist ein schwerwiegendes Bedenken.

\section{Was macht den Abhang rutschig?}

Der Einwand der Nichtabgrenzbarkeit vom reproduktiven Klonen kennt mehrere Varianten. Betrachten wir sie genauer: Eine Gruppe von Experten der EnquêteKommission des deutschen Bundestages «Recht und Ethik der modernen Medizin" formulierte es so:

«Dieselbe Technologie [...] kann auch für das reproduktive Klonen verwendet werden [...]. Jede Forschung zur Verbesserung des itherapeutischen Klonens» rückt daher das (reproduktive Klonen), das bisher von niemandem politisch gewollt wird und das ethisch nicht gerechtfertigt werden kann, einen Schritt näher an den Bereich des Machbaren heran." [2].

Der Einwand ist der, dass mit der Erforschung und Entwicklung des therapeutischen Klonens die Kerntransfertechnologie beim Menschen entwickelt wird, die dann ohne weiteres auch für das reproduktive Klonen zur Verfügung steht. Der "Abhang" ist rutschig, weil beide Konzepte von Klonen dieselbe Technik benötigen. Man kann nicht nur die Technik für das eine entwickeln, ohne auch das andere möglich zu machen.

Dies ist zweifellos richtig. Aber es bezieht sich nur auf die technische Machbarkeit. Als solches wäre dieser auf die Technologie bezogene "slippery slope»Einwand auf viele biomedizinische (und andere) Technologien zu beziehen, wie z.B. die somatische Gentherapie. Auch dort rückt mit den Techniken zur genetischen Manipulation somatischer Zellen die Keimbahnmanipulation näher. Trotzdem hat man das dort nicht zum Anlass genommen, die somatische Gentherapie zu verbieten. Die Antwort war vielmehr die, das eine klar zu umschreiben und zu verbieten, und das andere unter Regeln zuzulassen. Entsprechend könnte man vielleicht auch im Fall des therapeutischen Klonens vorgehen. Technische Machbarkeit schliesst ein rechtliches Verbot nicht aus.

Eine zweite Variante: Der Genetiker Axel Kahn, Mitglied des Französischen Comité consultatif national d'éthique gibt dem "slippery slope»-Argument folgende Richtung:

"Lorsque des milliers de tels embryons seront obtenus pour préparer des populations cellulaires, il ne suffira plus que d'en transplanter certains dans des utérus pour, peut-être, conduire à la naissance de bébés clonés.» [3] 
Hier ist der Einwand der, dass die Existenz klonierter Embryonen eine neue Situation schafft. Es können Versuche unternommen werden, gewisse dieser Embryonen vor der vorgesehenen Zerstörung zu retten, indem sie in den Uterus einer Frau mit Kinderwunsch übertragen werden. Die Wahrscheinlichkeit würde erhöht, dass trotz Verbot bald das erste geklonte Baby schreien wird.

Auch diese Überlegung ist zweifellos ernst zu nehmen: Wenn geklonte Embryos einmal in vitro da sind, könnte die Versuchung entstehen, sie doch in utero auszutragen. Motive könnten Sensationsgier oder eben das Retten des Kerntransferembryos vor seiner Zerstörung sein. Das zweite Motiv wird von der vorherrschenden Auffassung unterstützt, die von der ethischen Gleichsetzung von Nukleustransferembryonen mit gezeugten Embryonen ausgeht. Sie spricht einem Nukleustransferembryo dann auch ein Recht auf Leben zu, wenn ein solches Recht den gezeugten Embryonen zugesprochen wird. Dabei wird aber von den hohen gesundheitlichen Risiken abgesehen, die auf einem durch Kerntransfer entstandenen Individuum lasten. Man könnte deshalb sagen, das Übertragen von Kerntransferembryonen in die Gebärmutter einer Frau sei ein ethisch gegenüber dem betroffenen Individuum unverantwortlicher Menschenversuch und müsse deshalb verboten bleiben. Die Antwort auch gegen diese Variante des «slippery slope»-Argumentes könnte also in einem strengen rechtlichen Verbot der Weiterentwicklung von Kerntransferembryonen über das Blastozystenstadium hinaus bestehen.

Eine dritte Variante des Einwands fehlender Abgrenzbarkeit formulierte die Church and Society Commission der Conference of European Churches (CEC):

"[I]t seems illogical to allow the creation of a cloned human embryo knowing full well one would have to destroy it on ethical grounds, because it was unethical to allow it to go to term to produce a cloned baby." [4]

Hier ist der Einwand von logischer Natur. Man kann nicht der Herstellung klonierter Embryonen zustimmen, wenn man schon weiss, dass es unethisch wäre, sie über ein bestimmtes Stadium hinaus sich entwickeln zu lassen. Eine menschliche Würde des Embryos kann nicht dadurch anerkannt werden, dass man es von vorneherein an der Entwicklung hindert. Die zitierte Formulierung gibt die Struktur des Arguments aber nicht ganz klar preis. Dietmar Mieth hat, was hier gemeint sein könnte, einmal die «Spaltung des Menschlichen" [5] genannt. Falls wir einem Embryo, der sich in ein Kind entwickeln wird, als ein Wesen mit einer eigenen moralischen Würde betrachten, warum vereiteln wir dann seine Lebensmöglichkeiten? Es besteht die Gefahr, hier eine Art Doppelmoral einzuführen: eine moralische Einteilung menschlicher Embryonen in eine Klasse, die Personen werden und als solche geschützt werden, und eine zweite Klasse von Embryonen, deren Existenz darin besteht, Ersatzteile für die Mitglieder der ersten Klasse zu werden. Die Entscheidung, welcher Embryo ein Mitglied der ersten und welcher ein Mitglied der zweiten Klasse ist, wird wohlverstanden von den erwachsenen Mitgliedern der ersten Klasse getroffen. Kurz: Man kann einen Embryo nicht ethisch schützenswert finden und ihn, nachdem man ihn hergestellt hat, aus ethischen Gründen schon in einem frühen Entwicklungsstadium töten. Die Logik verlangt nach dem Gewährenlassen seiner Entwicklung, ja sogar nach der Verbesserung seiner Lebensbedingungen. Deshalb würde die Erlaubnis des therapeutischen Klonens aus normenlogischen Gründen früher oder später die Erlaubnis des reproduktiven Klonens nahelegen.

Auch diesem Einwand wohnt prima facie eine hohe Plausibilität inne. Aber nur unter der Voraussetzung, dass es nicht zusätzliche und unabhängige ethische Gründe gibt, die das reproduktive Klonen verbieten. Falls es solche gibt (ich glaube, dass es sie tatsächlich gibt; die beiden nächsten Teile dieser Folge handeln davon), wäre ein Verbot des reproduktiven Klonens u.U. doch mit der Anerkennung einer besonderen Schutzwürdigkeit jedes menschlichen Embryos, auch der klonierten, logisch kompatibel.

\section{Voraussetzungen dieser Einwände}

Alle drei Varianten des "slippery slope»-Einwandes besagen, dass die Zulassung und Entwicklung des therapeutischen Klonens unweigerlich zur Realität des reproduktiven Klonens führen wird. Damit dies jedoch überhaupt ein Einwand gegen die Erlaubnis des therapeutischen Klonens sein kann, müssen, so scheint mir, drei Voraussetzungen erfüllt sein:

1. Das reproduktive Klonen ist in jedem Fall ethisch verwerflich.

2. Es bestehen keine anderen Mittel, um das Klonen von Kindern wirksam zu verhindern.

3. Das Entstehen eines geklonten Kindes stellt einen so einschneidenden Verstoss gegen die Prinzipien der Menschenwürde dar, dass dies in keinem Fall aufgrund einer gesundheitspolitischen Güterabwägung als Resultat kriminellen Verhaltens in Kauf genommen werden darf.

Der erste Punkt bedarf der Klärung, kann sich aber auf einen breiten und überwiegenden Konsens stützen. Den zweiten Punkt habe ich oben bereits in Zweifel gezogen. Der Tatbestand des reproduktiven Klonens scheint mir jedenfalls hinreichend klar beschreibbar, um strafrechtlich verboten werden (oder bleiben) zu können, auch wenn das therapeutische Klonen zugelassen würde. Man müsse die Einsetzung eines geklonten Embryos in den Uterus einer Frau verbieten, die Entwicklung über das Blastozystenstadium hinaus ausschliessen, und die Entwicklung geklonter Embryonen an strenge medizinische Indikationen knüpfen.

Meine Unsicherheit bezieht sich vor allem auf den letzten Punkt. Auch wenn reproduktives Klonen generell für ethisch verwerflich gehalten wird (ich 
werde selbst in dieser Richtung argumentieren), und wenn es keine wirksamen Mittel gibt, um das Entstehen eines geklonten Kindes wirksam zu verhindern, bleiben zwei Fragen offen:

Ist das erstens mit einem Verbot des therapeutischen Klonens tatsächlich zu verhindern? Ist denn das Verbot des therapeutischen Klonens, um das wir hier diskutieren, ein wirksames Mittel, um die Geburt eines geklonten Babys zu verhindern, wenn gleichzeitig die Technologie des reproduktiven Klonens in Tierexperimenten so weit entwickelt ist, und nicht alle Länder der Erde Klonverbote statuiert haben?

Ist zweitens das Gezeugtsein durch Kerntransfer (statt durch Gametenfusion) als solches eine Schädigung des Kindes, eine Verletzung seiner Menschenwürde? Oder entsteht die moralische Verwerflichkeit des reproduktiven Klonens nicht vielmehr aus den einengenden sozialen Erwartungen (ähnlich werden zu müssen, wie die Person, von der der Zellkern stammt) an das Kind, wenn man von ihm weiss, dass es geklont, d.h. "der Klon ist von ...»? Könnte man diese sozialen Erwartungen durch bessere Aufklärung über die tatsächliche Rolle der DNA für die Individualität eines Menschen mildern? Trägt sogar eine rigoristische Verbotshaltung gegenüber jeder Form des Klonens zu einer Stigmatisierung illegal erzeugter geklonter Kinder in negativer Weise bei?

Aus diesen Gründen scheint mir das «slippery slope»-Argument nicht genügend klar zu sein, um eine eingehende ethische Auseinandersetzung mit dem therapeutischen Klonen mit prinzipiell offenem Ausgang von vorneherein überflüssig zu machen.

\section{Weitere offene Fragen}

Woher kommen die Eizellen?

Das reproduktive Klonen wäre mit der Verwendung mindestens einer menschlichen Eizelle verbunden. Diese Eizellen könnten entweder aus In-vitro-Fertilisationen stammen, wo mehr Eizellen gewonnen als zur Befruchtung verwendet werden können. Diese Quelle ist zweifellos zahlenmässig begrenzt, könnte aber für die Phase der Forschung und für besonders dringende Anwendungen genügen. Die Eier dürften selbstverständlich nur verwendet werden, wenn die Spenderin dazu frei und informiert zugestimmt hat. Der Gesetzgeber könnte den Gebrauch von Eizellen auf diejenigen einschränken, die in der fortpflanzungsmedizinischen Praxis ohnehin anfallen und bei denen die Zustimmung der Frauen vorliegt. Damit würde automatisch auch das therapeutische Klonen quantitativ begrenzt. Eizellen blieben ein knappes Gut, die mit Bedacht, nur für die besonders dringenden Fälle verwendet werden können.

Oder der Gesetzgeber könnte auch die Eizellspende zum Zweck des therapeutischen Klonens zulassen. Die Überlegungen, die ihn im Fortpflanzungsmedizingesetz (Art. 4) bewogen haben, die Eispende zu Fortpflanzungszwecken zu verbieten, müssten die Eispende zu therapeutischen Zwecken nicht unbedingt ausschliessen. Denn dort standen Argumente zum besonderen Status der Mutterschaft (im Vergleich zur Vaterschaft) im Vordergrund [6]. Aber die Befürchtung, auf Frauen könnte Druck ausgeübt werden, würde auch hier gelten. Die Eispende ist mit einem körperlichen Eingriff verbunden, der belastend ist und auch Risiken für die Spenderinnen beinhaltet [2]. Ein System von positiven Anreizen (z. B. Eizellen gegen Geld) wäre ethisch problematisch. Denn es besteht die Gefahr einer neuen strukturellen Ausbeutung des Körpers finanziell bedürftiger Frauen, die dann durch Eispende Geld verdienen könnten. Vergleiche mit der Prostitution drängen sich auf.

\section{Weshalb keine tierischen Eizellen?}

Eine (hypothetische) technische Alternative zur Verwendung menschlicher Eizellen wären tierische Eizellen [7]. Gesetzt, ein solches Verfahren könnte so weit entwickelt werden, dass die hergestellten Stammzellen eine ausreichende Biosicherheit bieten: Wäre es überhaupt ethisch legitim, durch Kerntransfer Tier-Mensch-Mischembryonen (nennen wir sie Nukleustransferchimären) zu erzeugen?

In Ländern, wo die Herstellung von menschlichen Embryonen zu Forschungszwecken verboten ist, wie es in vielen europäischen Ländern, einschliesslich der Schweiz und Deutschland, aber ausschliesslich UK, der Fall ist, wären Nukleustransferchimären ein möglicher Ausweg. Sowohl das Deutsche Embryonenschutzgesetz als auch das Schweizerische FMedG könnten hierfür eine Lücke bieten. Für die Schweiz ist der Art 36 FMedG relevant, wo die Herstellung von Chimären oder Hybriden verboten wird. Dieses Verbot richtet sich eindeutig gegen die Herstellung lebensfähiger Mischorganismen aus menschlichen und tierischen Teilen, aber womöglich nicht gegen die Herstellung "gemischter" Blastozysten, die in Zellkulturen aufgelöst werden. Zusätzlich ist nach Art 2m FMedG die Chimärenbildung so definiert: «die Vereinigung totipotenter Zellen aus zwei oder mehreren genetisch unterschiedlichen Embryonen zu einem Zellverband.» - Diese Chimärendefinition wird von der tierischen entkernten Eizelle mit menschlichem Zellkern jedenfalls nicht erfüllt.

\section{Reprogrammierung bis zur Totipotenz?}

Verschiedene Kritiker des therapeutischen Klonens empfehlen die Reprogrammierung adulter Stammzellen als technische Alternative. Zum Beispiel die Pontifikale Akademie für das Leben: «The possibility, now confirmed, of using adult stem cells to attain the same goals as would be sought with embryonic stem cells - even if many further steps in both areas are necessary before clear and conclusive results are obtained - indicates that adult stem cells represent a more reasonable and human method for making correct and sound progress in this new field of research and in the therapeutic applications which it promises. These applications are undoubtedly a source of great hope for a significant number of suffering people» [8]. Die jüngsten wissenschaftlichen Erfolge lassen tatsächlich aufhorchen [9]. Man kann Stammzellen des einen Typs (z.B. eine Blutstammzelle) in Stamm- 
zellen anderer Typen (z.B. eine Nervenstammzelle) umwandeln. Dabei zeigt sich (mit technischer Hilfe) zumindest eine Pluripotenz von adulten Stammzellen, also von Zellen, die dem Körper des Patienten entnommen werden können. Wenn wir uns nun eine kleine Spekulation erlauben: Was wäre, wenn es sich herausstellen würde, dass sich eine adulte Stammzelle so weit "zurückprogrammieren" lässt, dass sie eine Art Blastozyste zu bilden fähig ist? Müsste man dann auch adulte Stammzellen prinzipiell für totipotent ansehen? Was wäre in ethischer Perspektive der Unterschied zwischen zur Totipotenz reprogrammierten "adulten" Stammzellen und Nukleustransferembryonen? Beide Konstrukte würden einen Zellkern eines lebenden, geborenen Menschen enthalten.

Müsste sich unser Körperverständnis grundlegend wandeln, wenn es sich herausstellt, dass auch bestimmte Körperzellen in die Lage gebracht werden können, neue Embryonen zu bilden? Müsste das Kriterium der Potentialität oder das Kriterium der Individualität, die heute dem besonderen Schutz von Embryonen zugrundeliegen, tiefgreifend überdacht werden? Hätte man überhaupt noch überzeugende Gründe in der Hand, das therapeutische Klonen zu verbieten, wenn eine Quasitotipotenz bis zur Blastozyste auch mit adulten Stammzellen möglich wäre? - Ich vermag diese Fragen heute nicht zu beantworten.

\section{Literatur}

1 Rehmann-Sutter C. Human Cloning? Teil 1: Der ethische Status von Nukleustransferembryonen. Anmerkungen zum "therapeutischen Klonen». Schweiz Ärztezeitung 2001; 82(19):983-6.

2 Experten der Enquête-Kommission. Verrohstofflichung der Gesellschaft. Gen-ethischer Informationsdienst GID 2001; 114:29-30.

3 Kahn A. Le clonage thérapeutique est-il légitime? Libération, 5 décembre 2000.

4 Church and Society Commission of the Conference of European Churches (CEC). Therapeutic Uses of Cloning and Embryonic Stem Cells. In: The European Group on Ethics in Science and New Technologies to the European Commission. Press Dossier. Adoption of an Opinion on Ethical Aspects of Human Stem Cell Research and Use. Paris, 14 November 2000. p. 161-76.

5 Mieth D. Probleme der Ethik der Biomedizin am Beispiel der Klonierungsdebatte. In: Ach JS. Brudermüller G, Runtenberg C (Hrsg.). Hello Dolly? Über das Klonen. Frankfurt a.M.: Suhrkamp; 1998. S. 156-74.

6 Botschaft des Schweizerischen Bundesrates über die Volksinitiative "Zum Schutz des Menschen vor Manipulation in der Fortpflanzungstechnologie (Initiative für menschenwürdige Fortpflanzung, FMF)" und zu einem Bundesgesetz über die medizinisch unterstützte Fortpflanzung (Fortpflanzungsmedizingesetz, FMedG) vom 26. Juni 1996, S. $50 f$.

7 Solter D. Embryonic Stem Cells and Cloning: A new era in human biology and medicine. In: Friele MB (ed.). Embryo Experimentation in Europe. Bio-medical, legal, and philosophical aspects. Grey Series Nr. 24. Bad NeuenahrAhrweiler: Europäische Akademie. 2001; p. 13-31.

8 Pontifical Academy for Life. Declaration on the Production and the Scientific and Therapeutic Use of Human Embryonic Stem Cells. In: The European Group on Ethics in Science and New Technologies to the European Commission. Press Dossier. Adoption of an Opinion on Ethical Aspects of Human Stem Cell Research and Use. Paris, 14 November 2000. p. 147-58.

9 Lovell-Badge R. Stem Cell Therapy: The Potential Importance of Research Into (Therapeutic Cloning), Embryonic Stem Cells and Adult Stem Cells. In: Friele MB (ed.). Embryo Experimentation in Europe. Bio-medical, legal, and philosophical aspects. Grey Series Nr. 24. Bad NeuenahrAhrweiler: Europäische Akademie; 2001. p. 32-57. 